\title{
Correspondence
}

\section{Beta-blockers and anesthetic preconditioning: friend or foe?}

To the Editor:

We read with interest the recently-published article by $\mathrm{Yu}$ and Beattie reporting a meta-analysis investigating the effects of volatile anesthetics on morbidity and mortality in patients undergoing coronary artery bypass graft surgery. ${ }^{1}$ It was reported that volatile anesthetics do not reduce mortality compared to $i v$ anesthesia, but reduce postoperative levels of troponin I as a marker of myocardial ischemic damage. Strikingly, patients receiving $i v$ anesthetics had a significantly higher incidence $(28 \%)$ of beta-blocker utilization compared to patients receiving volatile anesthetics. This is an extraordinarily important finding. Yu and Beattie conclude that "some myocardial protective effects of the inhalation anesthetics may have been counteracted as beta-blocker utilization was unequally distributed...”. We agree with this conclusion insofar as the disproportion of beta-blocker use may have influenced the results. However, we surmise that concurrent beta-blocker therapy inhibited the cardioprotective effects of volatile anesthetics and that the beneficial effects of the volatile anesthetics would have been even more pronounced, if no beta blockers had been used at all. This contention is derived from the fact that volatile anesthetic preconditioning is mediated by beta-adrenergic signalling. Unspecific blockade of beta-adrenergic receptors abrogates desflurane-induced preconditioning in isolated human atrial myocardium. ${ }^{2}$ Furthermore, volatile anesthetic preconditioning is abolished by concurrent blockade of beta ${ }_{1}$-adrenergic receptors by esmolol and downstream protein kinase A by the selective blocker H-89 in the rabbit heart in vivo. ${ }^{3}$ This result can also be obtained using the beta ${ }_{1}$ selective blocker metoprolol. ${ }^{4}$

Perioperative beta-blocker therapy is recommended by the American College of Cardiology/American Heart Association in cardiac high risk patients. However, evidence is emerging that the beneficial effects of perioperative beta-blocker therapy are less impressive than originally assumed. ${ }^{5}$ Patients at low cardiac risk might even be harmed by inadvertent bradycardia and hypotension. ${ }^{6}$ Thus, novel cardioprotec- tive strategies need to be considered that are of equal benefit to low and high risk patients. Volatile anesthetic preconditioning is such a promising new strategy in perioperative cardioprotection. However, interactions with beta-blockers and other cardiovascular medications, potentially abrogating preconditioning need to be considered when anesthetic preconditioning is to be utilized for cardioprotection.

The experimental data and this interesting finding of the meta-analysis suggest that volatile anesthetic preconditioning and beta-blocker therapy are incompatible cardioprotective strategies. This might, among other factors, explain the limited effects of volatile anesthetics on mortality and perioperative myocardial infarction in certain patient populations. Further studies are needed to investigate the interactions between cardiovascular medications and volatile anesthetic preconditioning.

Markus Lange MD

Norbert Roewer MD PhD

Franz Kehl MD PhD DEAA

Universität Würzburg, Würzburg, Germany

E-mail: franz.kehl@mail.uni-wuerzburg.de

Accepted for publication January 3, 2007.

\section{References}

$1 \Upsilon_{u} \mathrm{CH}$, Beattie WS. The effects of volatile anesthetics on cardiac ischemic complications and mortality in CABG: a meta-analysis. Can J Anesth 2006; 53: 906-18.

2 Hanouz JL, Yvon A, Massetti M, et al. Mechanisms of desflurane-induced preconditioning in isolated human right atria in vitro. Anesthesiology 2002; 97: 33-41.

3 Lange M, Smul TM, Blomeyer CA, et al. Role of the betal-adrenergic pathway in anesthetic and ischemic preconditioning against myocardial infarction in the rabbit heart in vivo. Anesthesiology 2006; 105: 50310.

4 Lange M, Smul T, Redel A, Roewer N, Kehl F. Coadministration of desflurane and metoprolol blocks anesthetic-induced preconditioning and cardioprotective effects of beta adrenergic blockade in the rabbit heart in vivo. Anesthesiology 2005; 103: A469 (abstract).

5 Yang H, Raymer K, Butler R, Parlow J, Roberts R. 
The effects of perioperative beta-blockade: results of the metoprolol after vascular surgery (MaVS) study, a randomized controlled trial. Am Heart J 2006; 152: 983-90.

6 Lindenauer PK, Pekow P, Wang K, Mamidi DK, Gutierrez B, Benjamin EM. Perioperative beta-blocker therapy and mortality after major noncardiac surgery. N Engl J Med 2005; 353: 349-61.

\section{Reply:}

We thank Drs. Lange, Roewer and Kebl for expressing interest in our recent paper, using our findings that beta blockers were unequally distributed between groups in our recent meta-analysis ${ }^{l}$ to highlight their recent investigations which suggest a negative interaction between beta blockers and ischemic preconditioning in vivo. ${ }^{2}$ Their findings are both interesting and intriguing, and come at a time when the efficacy of perioperative beta blockade has become increasingly controversial. The evidence cited refers to beta blockade in non-cardiac investigations, an important distinction since our report was limited to cardiac surgery. Coronary artery surgery has definitive periods of ischemia and reperfusion, a situation which is infrequent and unpredictable in noncardiac surgery. Secondly, we emphasize, that unlike beta blockade, there is no clinical evidence of an ischemic preconditioning like the effect of inhaled anesthetics in non-cardiac surgery.

Interestingly, there is clinical evidence that contradicts Dr. Lange et al.'s supposition, information which we were originally unable to report due to space constraints. Seventy-nine percent of the patients in the DeHert et al. studies (cited in our meta-analysis) were concomitantly using beta blockers, equally distributed between iv and inhaled anesthesia. (odds ratio 0.98; 95\% confidence interval 59-1.64 P=0.95). In these predominately beta blocked patients less troponin was released postoperatively in patients who received anesthesia with sevoflurane (weighted mean difference -2.95 ; 95\% confidence intervals $-3.3,-2.6 P=<0.000001)$. These results suggest that ischemic-like preconditioning occurs in the presence of clinically beta-blocked patients.

This new controversy highlights the immediate need for safety and efficacy studies on combination therapies and drug interactions in both cardiac and non-cardiac surgery. However, on the available evidence it is premature to conclude that anesthetic preconditioning and beta blockers are incompatible.
W. Scott Beattie MD PhD FRCPC

Chun Hua Yu MD

University Health Network, University of Toronto, Toronto, Canada

E-mail: scott.beattie@uhn.on.ca

\section{References}

$1 \Upsilon_{u} \mathrm{CH}$, Beattie WS. The effects of volatile anesthetics on cardiac ischemic complications and mortality in CABG: a meta-analysis. Can J Anesth 2006; 53: 906-18.

2 Lange M, Smul TM, Blomeyer CA, et al. Role of the beta 1 -adrenergic pathway in anesthetic and ischemic preconditioning against myocardial infarction in the rabbit heart in vivo. Anesthesiology 2006; 105: 50310.

\section{Use of the "Aretube" to facilitate ventilation during percutaneous tracheostomy}

To the Editor:

During percutaneous tracheostomy, the endotracheal tube is usually withdrawn to a sub-glottic position, or replaced by a laryngeal mask airway. ${ }^{1}$ This procedure has disadvantages and risks including: ${ }^{2}$ air leaks with hypercarbia, hypoxia, bronchial inhalation, and accidental extubation. ${ }^{3,4} \mathrm{~A}$ new airway device, the "Aretube" (Figure) has been developed recently to address some of these difficulties. The Aretube provides ventilatory assistance through intubation limited to one part of the larynx. The Aretube has a proximal orifice, and a distal orifice with two cuffs, the first of which has a $20-\mathrm{mL}$ capacity, situated $1 \mathrm{~cm}$ from the distal end. The second cuff has a $100-\mathrm{mL}$ cacacity and is situated approximately $\mathrm{l} \mathrm{cm}$ proximal to the first. For correct positioning, the first cuff is placed in a supraglottic position immediately above the vocal cords, while the second cuff (once inflated) is sited in the oropharynx, thus fixing the device while applying gentle pressure on the first cuff. When inflated, the first cuff exerts pressure around the glottis, ensuring a good seal.

The Aretube combines two types of tubes, an 8$\mathrm{mm}$ internal diameter (ID) endotracheal tube and a disposable 4-mm ID laryngeal tube. The device is applied with the patient anesthetized and paralyzed. An airway exchange catheter is first advanced through the lumen of the existing tracheal tube, which is then removed. This tube exchange must be done with due consideration for the possibility of difficult reintubation. The patient's trachea is reintubated over the 